\title{
Normalization and Personalization of Learning Situations: NPLS
}

\author{
doi:10.3991/ijet.v4i2.818 \\ Mounia ABIK and Rachida AJHOUN \\ ${ }^{1}$ National Superior School of Computer Science and Systems Analysis (ENSIAS), Rabat, Morocco
}

\begin{abstract}
The personalization of learning is a major pedagogical challenge. There are several projects involving the production of personalizable learning platforms such as Reload-LDE and Alfanet. The objective of these projects is to produce new standardized and personalizable learning situations. However, on the Web, an important number of learning situations exist. These situations are rich in information, but do not consider all the individual characteristics of participants who take part in the learning experience, nor their technical environments. This paper presents a help system that can transform an existing learning situation to a more standardized and personalizable structure, depending on the specific learning context.
\end{abstract}

Index Terms - Context of learning personalization, learning situation, normalization, personalization.

\section{INTRODUCTION}

With the development of the TIC, pedagogues have relied on distance learning to improve the quality of learning. Indeed, while eliminating spatiotemporal constraints, learning can now be personalized according to specific learner profiles (prerequisite, objectives, etc.) assuring more effective learning experiences that better meet the course objectives. However, with the growth of distance learning (relative to the technological development), other technical challenges (accessibility, sharing of information...) have become primordial. This has pushed researchers to unite their efforts to face them.

Recently, although much research has been carried out concerning technical challenges, personalization remains a fertile field of research. Several online learning situations ${ }^{1}$ (course, seminary...) exist, but the quality of learning sometimes remains in question. Most of these learning situations, generally rich in information, do not take into consideration different individual learning strategies and styles and do not encourage learners to continue learning.

To overcome technical challenges (access, reuse, etc.) and educational challenges (personalization, collaboration, etc.), several standards concerning the structuring of courses (SCORM, IMS-LD, etc) and the profiles (IMSLIP, CC/PP...) exist. Authors can adopt these standards to produce new learning situations that comply with the pedagogues' expectations. Yet, considering the large investment in time and money to produce new learning situations and the quantity and the diversity of the available learning situations on the Web (rich in matter but

\footnotetext{
${ }^{1}$ Learning situation is a set of conditions and events able to lead a person to build knowledge.
}

does not deal the need for personalization), the question is how could to improve the existing learning experiences so that they better teacher expectations.

The objective of our work is twofold. First of all, it aims at using existing learning situation by standardizing them in order to assure their use by any Learning Management System (LMS), as well as their exchange between users with different profiles (learner profile, group profile, terminal profile, network profile). Furthermore, our work intends to render those objects personalizable to better meet specific learning contexts.

In this work, we present our vision of personalizing learning by identifying the most relevant personalization criteria. Thereafter, we present our approach to normalize and personalize learning situations that we have called NPLS (Normalization and Personalization of the Learning Situations). The result of this approach is a help system destined to the authors of online learning situations.

\section{History of the Personalization of Remote LEARNING}

Currently, in a society based on competence, the objective of learning is to help the learner learn [4] [5]. All educational work is to emphasize the relation of the learners to the knowledge by helping them build their own representation of knowledge according to their acquirements and experiences. Nonetheless, since classical learning remains largely fails to achieve this objective, educators and computer specialists have joined their efforts so that distance learning can rise to this challenge. However, with the development of distance learning and the technological evolution, the sphere of personalization is enlarged.

Indeed, in the beginning, with the use of correspondence courses, distance learning attempted to personalize learning according to the learner's rhythm and the language learners mastered, based on behaviorism. Since then, with the apparition of e-learning, personalization has taken other dimensions. In fact, with Internet as an environment of interaction and communication, distance learning has become learner centered, based on constructivist learning theory. Constructivist theory requires a more dynamic interaction between learners and the LMS and is based on the learner's mental schema. Constructivist methodologies encourage interactions (synchronous or asynchronous) between the learners in collaborative environments. Accordingly, for this type of learning, in addition to learner characteristics (learner or group of learners), personalization must take into account the technical environment, including network bandwidth.

Recently, with the use of the mobile in learning, other personalization factors impose themselves relative to net- 
works (GPRS, UMTS, etc.) and to terminals (PDA, PCtablet, etc.) used to assist learning.

Consequently, learning personalization depends on several factors relative to the learner's profile, to the group's profile, in case of collaborative learning, and to the technical environment's profile (network profile, terminal profile, etc.). These profiles constitute the context of personalization for learning. This context is the key to successfully personalizing learner interaction with content.

\section{CONTEXT OF LEARNING PERSONALIZATION}

Context is a multidisciplinary concept and many definitions in several areas such as computer science and psychology exist [6]. These definitions are either very abstract (which makes the formalization of the context very difficult), or very specific to a particular area. The definition of context which is the most widely used is given by Dey [7] who writes that "Context is any information that can be used to characterize the situation of entities (i.e. whether a person, place or object) that are considered relevant to the interaction between a user and an application, including the user and the application themselves."

From this definition and because of the need to personalize learning situations we define the context as follows: the entity represents learning and the context covers all information that characterizes the situation "personalization" of the learning entity. This information is related to the participant taking part in the learning (learner, group of learners) and the technical environment (network and terminal) [1]. Accordingly, the three major components called profiles constitute the context of learning personalization shown in Figure 1.

- The participant profile: A participant in learning is either a learner or a group of learners. Consequently, two types of profiles exist:

o The learner profile that gathers information about the learner, such as his learning style, preferences, knowledge, etc.

o The group profile that gathers information about the group of learners such as group preferences and the type of group interaction.

- The terminal profile: There is currently a wide variety of devices such as computers, mobile phones, PDA, Smart Phones, etc. Each type of these devices has its own characteristics (the screen size, memory size, browser, etc.) These characteristics are reflected in a model called "terminal profile".

- The network profile: Today, communication networks are diverse (RTS, WIFI, GPS, GPRS, UMTS etc.). Each network has its own characteristics (bandwidth, delay, gigue, etc.). These can be incorporated into a model called "network profile." In the rest of this article, the network profile will be ignored and our research will focus on the participant profile and the terminal profile.

\section{A. Personalization criteria of the learner profile}

The individual differences of each learner are determined from the information related to cognitive function. Several researches are concerned with the expression of this information [8] [9].

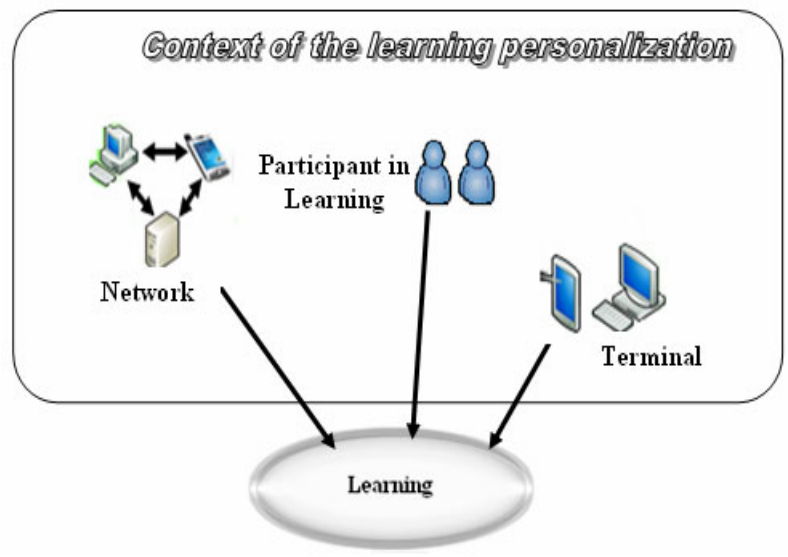

Figure 1. Context of learning personalization.

From these researches, we have chosen the information which constitutes the most relevant personalization criteria:

- The personal information of the learner such as age, sex, etc. This information is gathered in a criterion called "Personal";

- The information relating to the learner socio-cultural contexts (culture, languages, etc.), to the device preferences (text, audio, video etc.), and to the human computer interface preferences (the color choice, fonts, etc.). This information is gathered by the criterion called "Preference ";

- The information concerning what the learner knows and does not know. For example, to know the functionality of a tool is knowledge about the tool. This information is gathered in a criterion called "Knowledge";

- The information related to the learner's past experiences which present the learner's know-how and the learner's familiarity with the system on which he/she will work. This includes, for example, work done using a specific tool represents the learner's experiences on this tool. This information is gathered in a criterion called "Experience ";

- The information not related to specific knowledge about or experiences with the domain. It is acquired by the learner's past experiences and is associated with other indispensable knowledge related to the operation of the system presented to the learner. For example, the expertise of a learner on a tool is not the sum of his/her knowledge about the functionality of the tool, rather on his/her ability to apply and combine these functionalities to indentify and solve problems. This information is gathered in a criterion called "Competence ";

- The information related to the whole of behaviors (or performances) that learner must be able to demonstrate after learning. This information is gathered in a criterion called "Objective";

\section{B. Personalization criteria of the group profile}

A group is a number of individuals (learners, teachers) gathered for a common goal (to do exercise together, exchange opinions on a given subject, etc.) during a given time. The interactions between the group members define 
the whole of the exchanges between them. They are influenced by the size, the attitudes, the remarks and the reactions of the group members. The interactions are structured and they develop with time [10] [11].The structuring of these interactions is based on the roles of the group members during the learning activity. Each group member has a role (learner, stimulator, assistant, manager, etc.), but the paramount role is that of the manager, also called the leader, because he/she is the guarantor of the group unit.

After an analysis of this group definition, we created two principal categories of criteria: the group constitution category and the communication environment category. These two categories contain personalization criteria of the group profile:

1) Personalization criteria of the group constitution category:

For a collaborative activity, how can we choose learners to constitute a homogeneous group of learners? In other words, what are the criteria of personalization which must be taken into consideration to construct a group of learners?

A group is a whole of learners with common objectives and prerequisites. So the criteria of personalization concerning the group constitution category are those pertaining to the intersection of the group profile and the learners profiles.

Therefore, the personalization criteria concerning the group constitution category are:

- Objective: the objective of the learning activity pursued by the group (resolution of a problem, acquirement of a technique, exchange of experience, etc.). The objective represents the reason for the group's existence and it relates the group type (working groups, discussion groups) [12] [13]. It is a criterion that necessarily belongs to the intersection of the learner profile with the group profile.

- Prerequisite: it is the set of knowledge and experiments required by the activity and which must be included in the intersection of the individual learner profiles of the individuals forming the group.

\section{2) Personalization criteria of the environment of communication category:}

The communication and interactions within the group aim at achieving a collaborative activity. A collaborative activity associates roles to the communicative environment to reach its objective. This environment can have different types of interaction (synchronous and asynchronous) and different communicative tools and services (forum, chat, audio conferencing...).

Personalizing the communicative environment of a collaborative activity depends on the use of the adequate type of communication and the adequate service or communication tool to accomplish the collaborative activity.

The personalization criteria concerning the communicative environment:

- Type of interaction: This is defined in the group profile and is a criterion which specifies whether the group prefers to communicate synchronously or asynchronously;
- Preferences of service and tools of communication: These criteria specify the preferences of the group with regards the service and communication tool learners prefer to accomplish a learning activity. It is related to the type of interaction chosen. Indeed, if, for example, students prefer asynchronous interaction, the group may prefer forums or e-mail, but if the students prefer asynchronous interaction, the group may prefer to chat online.

\section{Personalization criteria of the terminal profile}

Online learning supposes access to content by learners using terminals. Recently, with technological development, a wide variety of devices that can play the role of terminals exist, including personal digital assistants (PDAs), laptops, mobile phones, etc. However, because these devices have different characteristics, it is necessary to adapt content according to the characteristics of these devices. In fact, because of the expanded use of mobile devices, adaptation to the device has become more and more important. This adaptation is based on the particular characteristics of each type of device. These characteristics are classified into two types of information:

- Hardware Information: the type of terminal (PC, PDA, mobile phone), mark, model, memory, audio characteristics (stereo, no sound), screen type, screen size, screen resolution screen, processor, etc.;

- Software Information: the operating system, operating system version, applications (identifying, name, version), audio format (name, format, standard, version), video format (name, format, standard, version), format pictures (name, format, standard, version), types of protocol.

\section{Correspondence Between Personalization CRITERIA AND STANDARDS}

In order to ensure the interoperability and the exchange between profiles, the need to standardize profiles is essential. Indeed, the greatest organizations which contribute to the elaboration of standards are:

- ISO/IEC, JTC1, SC36 and the IMS Learning Consortium for the normalization of participant profiles;

- $\mathrm{W} 3 \mathrm{C}$ for the normalization of the terminal profile

According to a comparative study that we did between ISO and IMS standards [2] [3], we were able to confirm that the work concerning profiles of both organisms meet our personalization needs. So, for us, the choice of the IMS or ISO model does not cause any problem. However, we noticed that the updates in IMS are frequent and access to information is easier compared to ISO / IEC JTC1/SC36 because the work of the latter is carried out by several working groups. Therefore, we opted to use IMS in this work.

The different correspondences between the personalization criteria that comprise the context of learning personalization and standardization are summarized as follows:

- Figure 2 displays the correspondence of personalization criteria relevant to the learner's profile with the IMS-LIP specification.

- Figure 3 represents the mapping of personalization criteria relevant to group profile with both the IMSLIP specification and IMS-Enterprise specification, 
according to the group constitution category and the category of the communication environment.

- Figure 4 presents the mapping of personalization criteria of the terminal profile with CC/PP specification.

According to the mapping presented below, all of the personalization criteria we have outlined are covered by already existing standardization work (IMS-LIP, IMSEnterprise, CC/PP). Consequently, for the conception of NPLS, we based our work on these standards to ensure the flexibility and the extensibility of our approach.

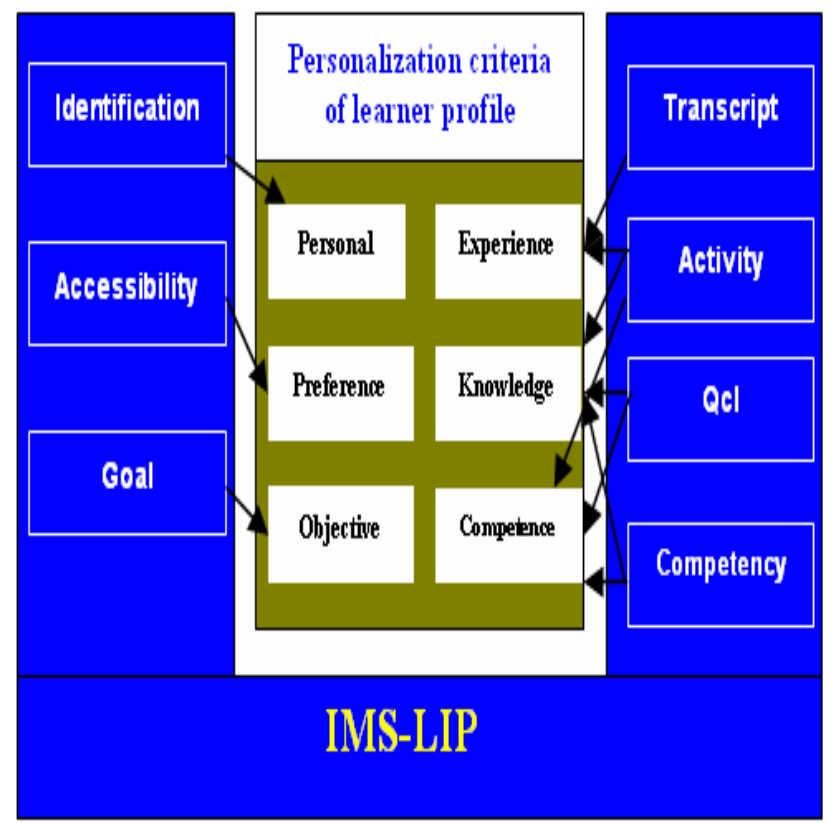

Figure 2. Mapping of the personalization criteria of the learner profile with IMS-LIP

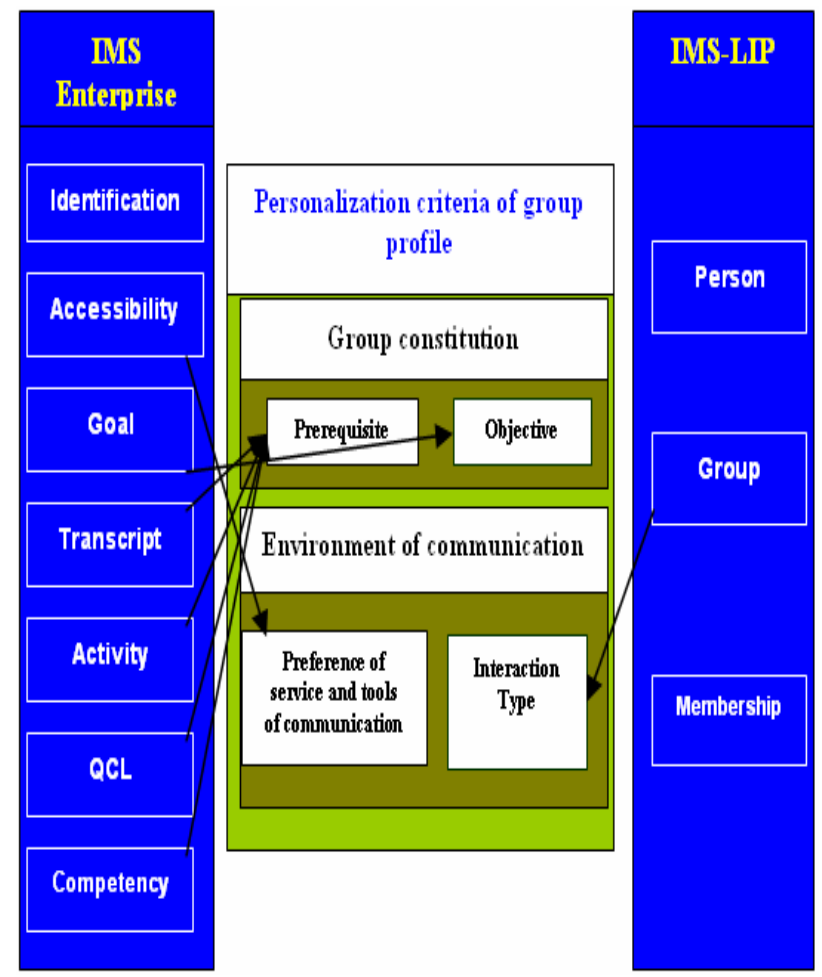

Figure 3. Mapping of the personalization criteria of the group profile with IMS-LIP et IMS-Enterprise

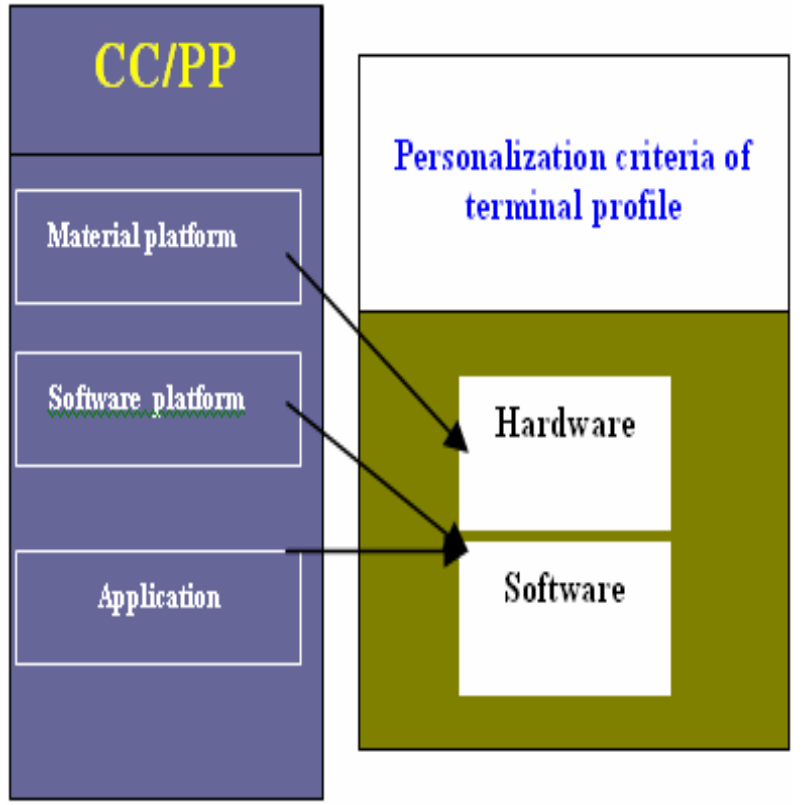

Figure 4. Mapping of the personalization criteria of the learner profile with IMS-LIP.

\section{THE FunCtional MODEL OF NPLS}

The main objective of our work is to propose a help system for designers of learning situations. This system transforms existing learning contexts that do not take learning personalization into account into systems that are more standardized and personalizable. The architecture of NPLS respects the following characteristics:

- Extensibility: the NPLS can integrate, at any instant, other profiles or other personalization criteria relative to the specific technology, thanks to the adoption of standards to define the context of learning personalization and personalization criteria.

- Modularity: The conception of the NPLS is supported by a modular architecture that facilitates its maintenance and assures its evolution. The elaboration of this architecture is based on the decomposition of independent modules that include the standardization module, the edition module and the personalization module.

- Reusability: the NPLS separates the content of the structure of the existing learning situations assuring the reuse of resources by other learners and the reuse of situations by any LMS.

- Genericity: the main objective of NPLS is the personalization that is based on the criteria belonging to the context of learning personalization. For the definition of these criteria, the NPLS depends on standards. This approach is characterized by its genericity compared with these standards for it can easily switch from one standard to another without influencing its components.

- Interoperability: the NPLS is destined for authors of learning situations, independently of any LMS. To ensure interoperability, we plan to publish it in the UDDI Registers and develop an access interface (WSDL) in order to employ the NPLS regardless of the platform. 


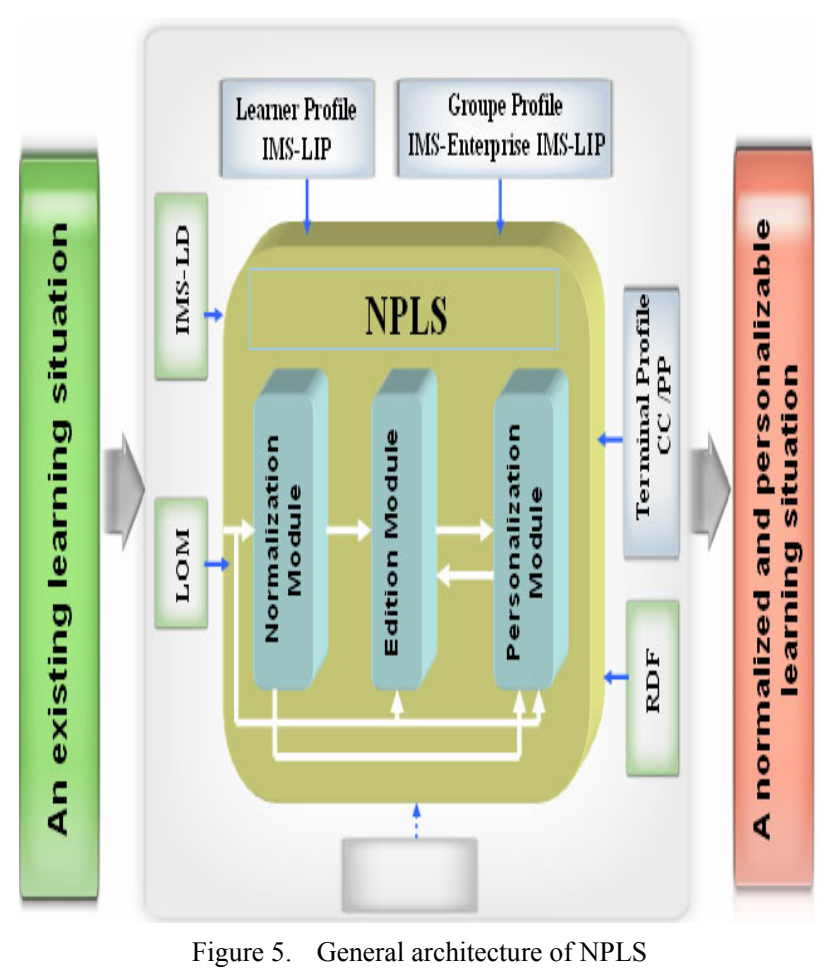

To make existing learning situations personalizable, we propose injecting personalization criteria to learning situations. This requires that these learning situations be structured and understood by the personalization module.

Hence, it is necessary to standardize the existing learning before the personalization phase. Therefore, we can identify two main modules: the module which is responsible for standardization and the module that is in charge of personalization. To be able to enrich structures emanating from the normalization module and personalization module (by the addition, for example, of activities or expressions of conditions), we add another module that edits learning situations. The use of this module can also concern the production of new standardized learning (cf. figure 5).

Accordingly, the functional architecture of NPLS is composed of three modules that are independent of each other:

\section{A. Normalization Module}

To ameliorate the quality of an existing learning situation and make it personalizable, it is necessary to standardize it in order to form a basic structure in which the author can insert personalization criteria concerning the individual or group learning situation.

Therefore, the interest of this module is to systematically transform an existing HTML structure (that is the most frequent format on the Web) to another structure that respects IMS-LD [10] by separating the content of form.

This module is composed of two elements: the "corrector" that transforms an HTML into an XHTML and the "IMS-LD generator", which transforms XHTML into an IMS-LD structure by extracting the resources that enable it to be used afterwards.

The result of the standardization module is an IMS-LD structure and a resource database shown in Figure 6.

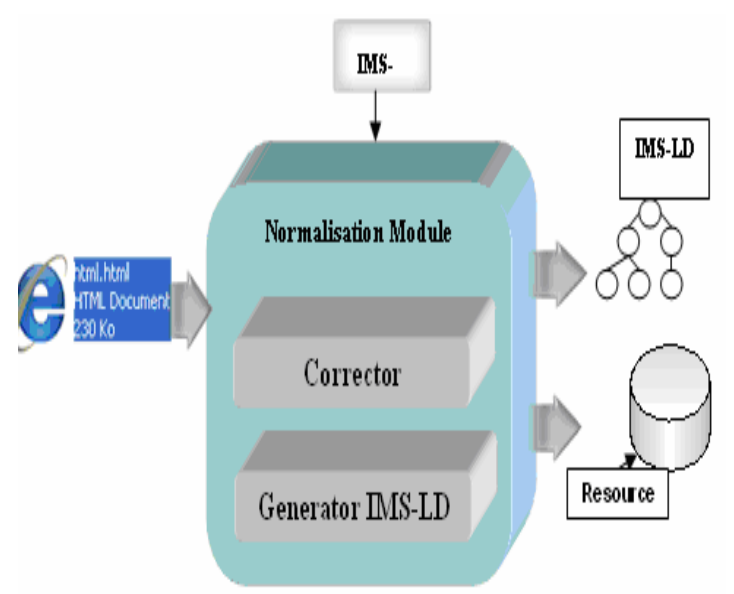

Figure 6. Normalization Module

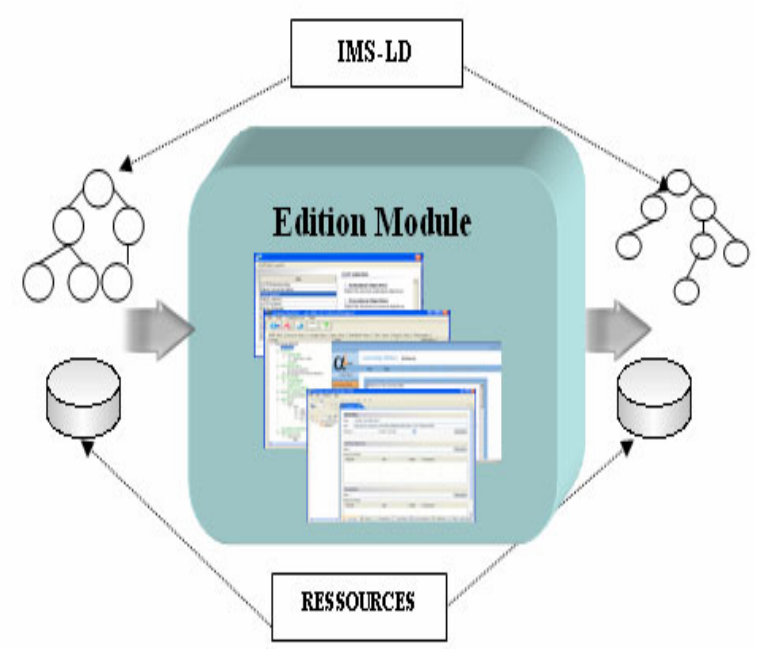

Figure 7. Edition Module

\section{B. Edition Module}

To be able to enrich the IMS-LD structure resulting from the normalization module (for example, by adding activities or expressions of conditions) or finalize the learning situation from the personalization module (by adding, for example, expressions of condition), we opted, instead of creating another editor, to insert an edition module of known editors such as Reload LDE and Collage. The result of the edition module is an IMS-LD structure which is enriched and a database of resources as shown in Figure 7.

NPLS allows for the addition or deletion of editors at any moment, assuring its openness to the preferences of the authors of the learning situations.

\section{Personalization Module}

This module represents the heart of our personalization approach and is responsible for providing to the author the personalization criteria to insert it in an IMS-LD structure. These criteria belong to the standardized profiles (IMSLIP, IMS-Enterprise, CC/PP...) used to establish the context of learning personalization.

So that the LMS can understand the meaning of these criteria and generate a specific learning situation according to the context of learning personalization, the person- 


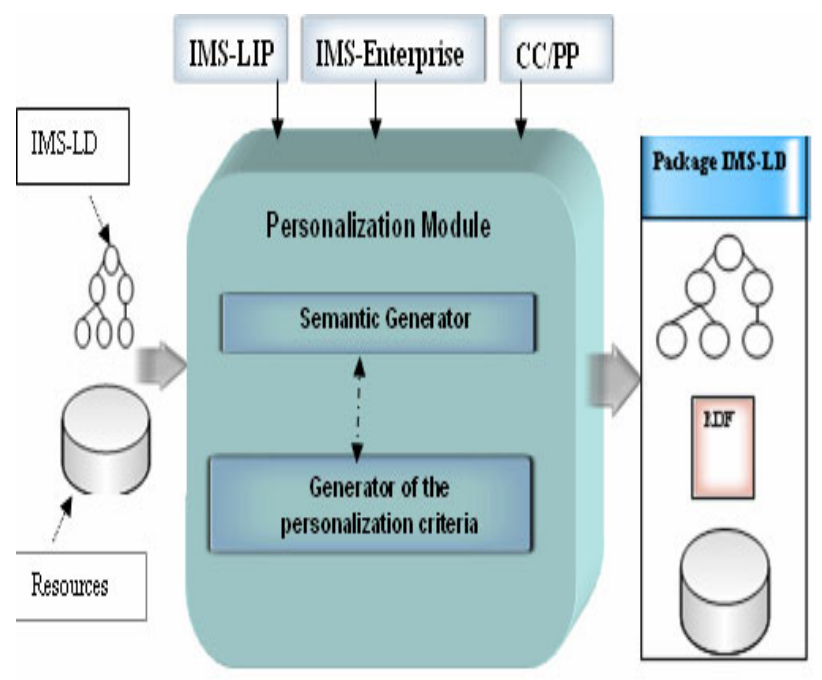

Figure 8. Personalization Module

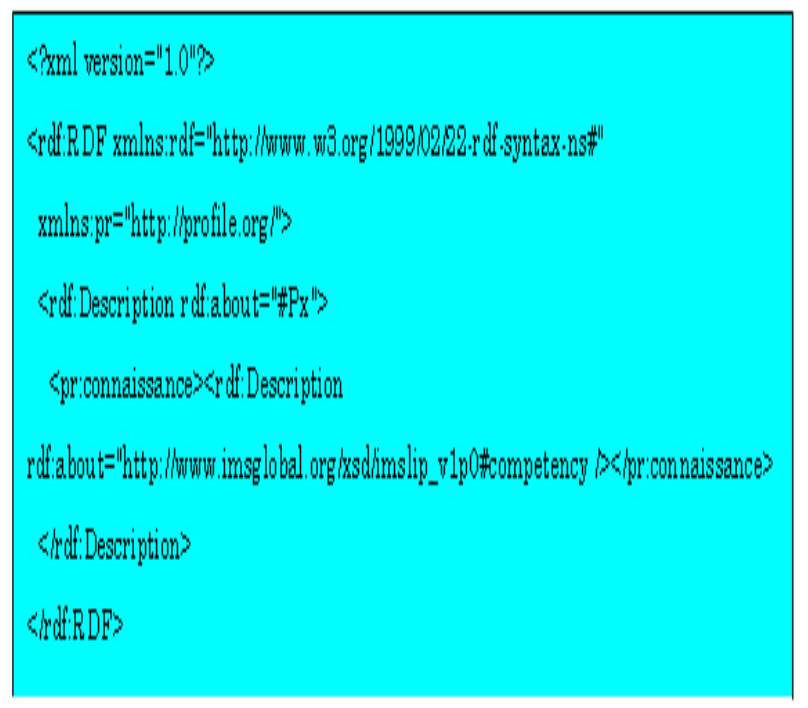

Figure 9. An example of RDF document describing the semantic properties in XML format

alization module produces a RDF document associated with the IMS-LD structure. This document describes relations between the personalization criteria inserted into the learning situation and the standardized profiles.

Therefore, the personalization module is composed of two main elements as shown in Figure 8.

- The personalization criteria generator: This element creates the personalization criteria to be inserted into the IMS-LD structure. The inserted criteria are from the different contexts of the learning personalization profiles, including the learner profile, the group profile and the terminal profile.

- The semantic generator: This element creates the personalization semantics criteria. Indeed, to assure the personalization of learning situations based on the inserted criteria, it is necessary for the LMS to understand the semantics of these criteria in order to access the concerned profiles. So, the role of the semantic generator is to establish links between criteria inserted into the IMS-LD structure and the corresponding categories of the standardized profiles (IMS-LIP, IMS-Enterprise, CC/ PP).
Figure 9 is an example of an RDF document generated by the personalization module that describes the semantics of a property "Px". It is knowledge presented in the "competency" of IMS-LIP, which can be located at http:/www.imsglobal.org/xsd/imslipv1p0.

\section{NPLS PROTOTYPE}

To validate our personalization approach, we have designed a prototype that validates the three NPLS modules. To ensure the extensibility of NPLS, the implementation of its interfaces is based on XML schemas. For NPLS's development and to its portability, we based our work on advanced technologies such as Java language that is secure, simple, robust and portable. We also used XML because it is independent of any platform. Figure 10 presents an example of NPLS interface.

\section{CONCLUSION}

With advanced technologies, the context of learning personalization evolves more and more by enlarging the sphere of personalization. Conscious of this, we propose an approach to normalize and personalize learning situations which is based on the context of learning personalization.

The originality of our work is that it improves the quality of the existing learning situations by making them standardized and personalizable. Our approach is distinguished by its flexibility. It permits, in case of need and at any moment, the addition or deletion of personalization criteria and standardized profiles that constitute the context of learning personalization. Besides, the semantics of the personalization criteria inserted assure the personalization of learning situations by any LMS.

To validate our approach, we have developed a prototype of the NPLS system using an environment based on Java and XML. The ultimate goal of this work is to identify and integrate NPLS into personalization criteria related to network profiles by taking into account the constraints of mobility and pervasivity.

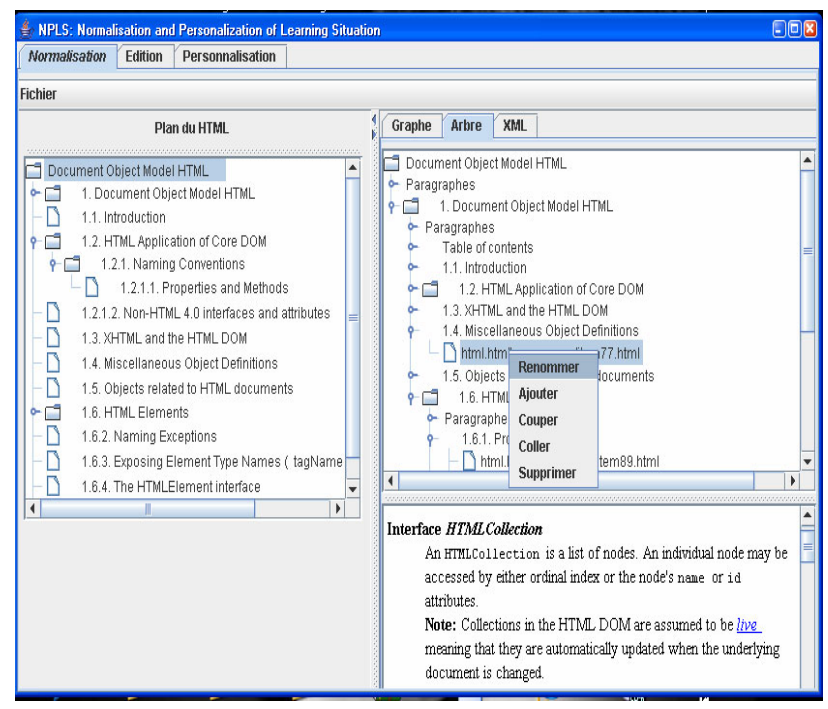

Figure 10. An example of an interface offered by the NPLS (normalization module) 


\section{ACKNOWLEDGMENT}

The authors wish to thank Ms. Hind Sidki for the validation of the English version of this document.

\section{REFERENCES}

[1] M. Abik, R. Ajhoun. "A Framework For Enabling Personalization Centred Learning Context". 5th Congress of Scientific Research Outlook and Technology Development in the Arab World "Scientific Innovation and Sustained Development”. October 26-30, Fez, Morocco, 2008.

[2] M. Abik, R. Ajhoun. "The Personalization of an individual and a group learning situation". IEEE Information and Communication Technologies International Symposium ICTIS'2007. ISBN: 9954877-0-2., April 3-5, Fes, Morocco, 2007, pp 51-54.

[3] M. Abik, R. Ajhoun. "Personalization of a group learning situation". Proceedings of the first International Conference on ICT \& Accessibility, pp. 181-186, Hammamet, Tunisia April 2007.

[4] M. Develay. "De l'apprentissage à l'enseignement". Paris : ESF édition, 1992, 163p.

[5] G., J. Pastiaux"Précis de pédagogie”. Paris : Nathan, 159p, 1997.

[6] M. Bazire, P. Brézillon. "Understanding Context Before Using it". Proceedings of the 5th International and Interdisciplinary Conference-Modeling and Using Context, Paris, 5-8 July, 2005, pp29-40.

[7] A. K. Dey, G. D. Abowd. "CybreMinder: A context-aware system for supporting reminders". Proceedings of the 2nd International Symposium on Handheld and Ubiquitous Computing (HUC2K), Heidelberg, Germany: Springer Verlag. 2000, pp 172-186.

[8] L. Razmerita, and G. Gouarderes...'Ontology-based User Modeling for Personalization of Grid Learning Services". In: Proceed- ings of Grid Services Workshop, ITS 2004, GLS 2004, Brazil, pp. 105-115.

[9] F.Mödritscher."The Impact of an E-Learning Strategy on Pedagogical Aspects". Journal of Instructional Technology \& Distance Learning (IDL), March, 2006. from http://www.campus02.at/ data/F-E paper moedritscher epedagogy 2006\%20(3).pdf

[10] F. Frasincar, G.J. Houben,'Hypermedia Presentation Adaptation on the Semantic Web". Proceedings of the 2nd International Conference on Adaptive Hypermedia and Adaptive Web-Based Systems, May 29-31, Malaga, Spain, 2002, pp. 133-142. from http://wwwis.win.tue.nl/ houben/respub/ah2002.pdf

[11] D. Beau "100 fiches de pédagogie des adultes à l'usage des formateurs".édition 1979 page 38.

[12] D.Anzieu et J-Y. Martin. "la dynamique des groupes restreints". livre, Editeur P.U.F 2003.

[13] J. Maisonneuve. "la dynamique des groupes", Editeur P.U.F, 2002.

\section{AUTHORS}

Mounia ABIK is with National Superior School of Computer Science and Systems Analysis (ENSIAS), Mohammed V Souissi University, Rabat, Morroco. E-mail: abmounia@gmail.com.

Rachida AJHOUN is with National Superior School of Computer Science and Systems Analysis (ENSIAS), Mohammed V Souissi University, Rabat, Morroco. E-mail: ajhoun@ensias.ma.

Manuscript received 30 January 2009. Published as submitted by the authors. 